\title{
On Faster Convergent Infinite Series
}

\author{
Dušan Holý, ${ }^{1}$ Ladislav Matejičcka, ${ }^{1}$ and L'udovít Pinda ${ }^{2}$ \\ ${ }^{1}$ Department of Physical Engineering of Materials, Faculty of Industrial Technologies in Púchov, \\ Trenčín University of Alexander Dubček in Trenčíin, I. Krasku 491/30, Púchov 02001, Slovakia \\ ${ }^{2}$ Faculty of Informatics, Univerzity of Economics, Dolnozemská 1, Bratislava 852 35, Slovakia
}

Correspondence should be addressed to Ladislav Matejíčka, matejicka@tnuni.sk

Received 12 July 2007; Revised 20 November 2007; Accepted 8 January 2008

Recommended by Laszlo Toth

Suffcient conditions, necessary conditions for faster convergent infinite series, faster $\tau$-convergent infinite series are studied. The faster convergence of infinite series of Kummer's type is proved.

Copyright (C) 2008 Dušan Holý et al. This is an open access article distributed under the Creative Commons Attribution License, which permits unrestricted use, distribution, and reproduction in any medium, provided the original work is properly cited.

\section{Introduction}

There have been papers devoted to the study of faster convergence of sequences. Certain methods concerning acceleration of convergence of sequences of partial sums of fixed series using linear or nonlinear transformations of partial sums of series are studied in [1]. The acceleration field of subsequence matrix transformations with respect to the convergence rate of the sequence being accelerated are studied in [2]. In [3], it is discussed a class of methods for summing sequences which are generalizations of a method due to Salzer [4], which accelerate some convergent sequences especially monotone sequences. In [5], is characterized the summability field of a matrix $A$ by showing $A$ is convergence preserving over the set of all sequences which converge faster than some fixed sequence $x, A$ is convergence preserving over the set of all sequences, or $A$ only preserves the limit of a set of constant sequences. Statistical acceleration convergence of sequences was discussed in [6]. The notion of faster convergent series with positive terms is defined in [7] and the notion of $\tau$-convergent series is defined in [8-10]. The statistical convergence of infinite series as a special case of $\tau$-convergence of infinite series is discussed in [11-14].

In this paper, some questions related to sufficient conditions and necessary conditions for faster convergent infinite series are studied, faster $\tau$-convergent series are defined and studied, and faster convergence of series of Kummer's type is proved. In [7, page 146], it is mentioned the Kummer's result: if $\sum_{n=1}^{\infty} a_{n}$ is convergent series with positive terms and with an unknown sum $a, \sum_{n=1}^{\infty} c_{n}$ is a convergent series with positive terms and with a known sum 
$c$, and $\lim _{n \rightarrow \infty} a_{n} / c_{n}=p>0$, then the Kummer series $\sum_{n=1}^{\infty} b_{n}$ has the same sum as $\sum_{n=1}^{\infty} a_{n}$ and is a faster convergent series than $\sum_{n=1}^{\infty} a_{n}$, if all terms of $\sum_{n=1}^{\infty} b_{n}$ are positive. Hence we can calculate the unknown sum $a$ faster by summation terms of the Kummer series $\sum_{n=1}^{\infty} b_{n}$, which is constructed by $\sum_{n=1}^{\infty} a_{n}, \sum_{n=1}^{\infty} c_{n}$, and $p$. In Lemma 4.1, we proved a faster convergence of the Kummer series $\sum_{n=1}^{\infty} b_{n}$ to the unknown sum $a$ of $\sum_{n=1}^{\infty} a_{n}$ without conditions of positivity of $p$ and terms of $\sum_{n=1}^{\infty} a_{n}, \sum_{n=1}^{\infty} b_{n}, \sum_{n=1}^{\infty} c_{n}$.

We denote by $\mathbb{N}$ the set of all positive integers and by $\mathbb{R}$ the set of all real numbers.

Definition 1.1 (see [2]). Let $\sum_{n=1}^{\infty} a_{n}, \sum_{n=1}^{\infty} b_{n}$ be convergent real series with the same sum, with nonzero terms and such that $b_{n}+b_{n+1}+\cdots \neq 0, n \in \mathbb{N}$. The series $\sum_{n=1}^{\infty} a_{n}$ is called faster convergent than $\sum_{n=1}^{\infty} b_{n}$ if $\lim _{n \rightarrow \infty}\left(a_{n}+a_{n+1}+\cdots\right) /\left(b_{n}+b_{n+1}+\cdots\right)=0$.

Lemma 1.2 (see [7]). Let $\sum_{n=1}^{\infty} a_{n}, \sum_{n=1}^{\infty} b_{n}$ be convergent real series with positive terms and with the same sum. If $\lim _{n \rightarrow \infty}\left(a_{n} / b_{n}\right)=0$, then $\lim _{n \rightarrow \infty}\left(a_{n}+a_{n+1}+\cdots\right) /\left(b_{n}+b_{n+1}+\cdots\right)=0$.

In what follows, we do not assume equality of sum of convergent series $\sum_{n=1}^{\infty} a_{n}, \sum_{n=1}^{\infty} b_{n}$ because we can construct series $\sum_{n=1}^{\infty} a_{n}^{*}, \sum_{n=1}^{\infty} b_{n}^{*}$, where $a_{n}^{*}=a_{n}$ and $b_{n}^{*}=b_{n}$ for $n \geq 2$ with the same sum.

\section{Faster convergent series}

Lemma 2.1. Let $\sum_{n=1}^{\infty} a_{n}, \sum_{n=1}^{\infty} b_{n}$ be convergent real series with positive terms. If

$$
\lim _{n \rightarrow \infty} \frac{a_{n}+a_{n+1}+\cdots}{b_{n}+b_{n+1}+\cdots}=0, \quad \text { then } \liminf _{n \rightarrow \infty} \frac{a_{n}}{b_{n}}=0 .
$$

Proof. By the way of contradiction, we suppose that $\liminf _{n \rightarrow \infty}\left(a_{n} / b_{n}\right)=q>0$, then there exists $n_{0} \in \mathbb{N}$ such that for every $n>n_{0}$ we have $0<q^{\star}<a_{n} / b_{n}$, where $0<q^{\star}<q$. From this follows $q^{\star}\left(b_{n}+b_{n+1}+\cdots\right)<\left(a_{n}+a_{n+1}+\cdots\right)$ for $n>n_{0}$, which is a contradiction with $\lim _{n \rightarrow \infty}\left(a_{n}+a_{n+1}+\cdots\right) /\left(b_{n}+b_{n+1}+\cdots\right)=0$, similarly for $q=\infty$.

Lemma 2.2. Let $\sum_{n=1}^{\infty} a_{n}, \sum_{n=1}^{\infty} b_{n}$ be convergent real series with positive terms. Let $\lim _{n \rightarrow \infty}\left(a_{n} / b_{n}\right)$ exist. Then

$$
\lim _{n \rightarrow \infty} \frac{a_{n}}{b_{n}}=0 \quad \text { iff } \lim _{n \rightarrow \infty} \frac{a_{n}+a_{n+1}+\cdots}{b_{n}+b_{n+1}+\cdots}=0 .
$$

The next example shows that under the conditions of Lemma 2.1, for all $0<r \leq \infty$, there exist a series $\sum_{n=1}^{\infty} a_{n}, \sum_{n=1}^{\infty} b_{n}$ such that $\lim _{\sup _{n \rightarrow \infty}}\left(a_{n} / b_{n}\right)=r$.

Example 2.3. Let $\left\{\alpha_{n} ; n \in \mathbb{N}\right\}$ be any sequence of real positive numbers which satisfy $\alpha_{n} \leq 1 / n^{4}$ for $n \geq 1$. We define the series $\sum_{n=1}^{\infty} a_{n}=r \alpha_{1}+\alpha_{1} / 1^{2}+r \alpha_{2}+\alpha_{2} / 2^{2}+\cdots+r \alpha_{k}+\alpha_{k} / k^{2}+\cdots$ and $\sum_{n=1}^{\infty} b_{n}=\alpha_{1}+1^{2} \alpha_{1}+\alpha_{2}+2^{2} \alpha_{2}+\cdots+\alpha_{k}+k^{2} \alpha_{k}+\cdots$. Then we have

$$
\begin{gathered}
\lim _{n \rightarrow \infty} \frac{a_{2 n-1}+a_{2 n}+\cdots}{b_{2 n-1}+b_{2 n}+\cdots}=\lim _{n \rightarrow \infty} \frac{r\left(\alpha_{n}+\alpha_{n+1}+\cdots\right)+\left(1 / n^{2}\right)\left(\alpha_{n}+\alpha_{n+1}+\cdots\right)}{\left(\alpha_{n}+\alpha_{n+1}+\cdots\right)+n^{2}\left(\alpha_{n}+\alpha_{n+1}+\cdots\right)}=0, \\
\lim _{n \rightarrow \infty} \frac{a_{2 n}+a_{2 n+1}+\cdots}{b_{2 n}+b_{2 n+1}+\cdots}=\lim _{n \rightarrow \infty} \frac{r\left(\alpha_{n+1}+\alpha_{n+2}+\cdots\right)+\left(1 / n^{2}\right)\left(\alpha_{n}+\alpha_{n+1}+\cdots\right)}{\left(\alpha_{n+1}+\alpha_{n+2}+\cdots\right)+n^{2}\left(\alpha_{n}+\alpha_{n+1}+\cdots\right)}=0,
\end{gathered}
$$


Dušan Holý et al.

$$
\lim _{n \rightarrow \infty} \frac{a_{2 n-1}}{b_{2 n-1}}=r, \quad \lim _{n \rightarrow \infty} \frac{a_{2 n}}{b_{2 n}}=0
$$

If $\sum_{n=1}^{\infty} a_{n}=\alpha_{1}+\alpha_{1} / 1^{2}+2 \alpha_{2}+\alpha_{2} / 2^{2}+\cdots+k \alpha_{k}+\alpha_{k} / k^{2}+\cdots$, then $\lim _{n \rightarrow \infty}\left(a_{2 n-1} / b_{2 n-1}\right)=\infty$.

Remark 2.4. It is evident that in general from $\lim _{n \rightarrow \infty}\left(a_{n}+a_{n+1}+\cdots\right) /\left(b_{n}+b_{n+1}+\cdots\right)=0$ does not follow $\liminf \operatorname{in}_{n \rightarrow \infty}\left(a_{n} / b_{n}\right)=0$. For example, if we put $a_{2 n}=b_{2 n}=1 /(n(n+1)), n \in \mathbb{N}$, and $a_{2 n-1}=-b_{2 n-1}=-1 /(n(n+1)), n \in \mathbb{N}$, we obtain $\lim _{n \rightarrow \infty}\left(a_{n}+a_{n+1}+\cdots\right) /\left(b_{n}+b_{n+1}+\cdots\right)=0$ and $\{-1,1\}$ is the set of all cluster points of the sequence $\left\{a_{n} / b_{n} ; n \in \mathbb{N}\right\}$.

In general, the condition $\lim _{n \rightarrow \infty}\left(a_{n} / b_{n}\right)=0$ does not imply the condition $\lim _{n \rightarrow \infty}\left(a_{n}+\right.$ $\left.a_{n+1}+\cdots\right) /\left(b_{n}+b_{n+1}+\cdots\right)=0$ as it follows from the next example.

Example 2.5. Let $\left\{\alpha_{n} ; n \in \mathbb{N}\right\}$ be a sequence such that $\alpha_{n}=q^{n}, n \in \mathbb{N}, 0<q<1 / 2$. We define convergent series $\sum_{n=1}^{\infty} a_{n}=\alpha_{1}+\alpha_{1}+\alpha_{2} / 2^{2}+\alpha_{2} / 2^{2}+\cdots+\alpha_{k} / k^{2}+\alpha_{k} / k^{2}+\cdots$ and $\sum_{n=1}^{\infty} b_{n}=$ $\alpha_{1}-\alpha_{1} / 2+\alpha_{2} / 3-\alpha_{2} / 4+\cdots+\alpha_{k} /(2 k-1)-\alpha_{k} / 2 k+\cdots$. From the definition, we obtain

$$
\begin{aligned}
b_{2 n}+b_{2 n+1}+\cdots & =\frac{-\alpha_{n}}{2 n}+\frac{\alpha_{n+1}}{(2 n+1)(2 n+2)}+\frac{\alpha_{n+2}}{(2 n+3)(2 n+4)}+\cdots \\
& <\frac{1}{2 n}\left(-\alpha+\alpha_{n+1}+\alpha_{n+2}+\cdots\right) \\
& =\frac{1}{2 n}\left(-q^{n}+q^{n+1}+q^{n+2}+\cdots\right)=\frac{q^{n}(2 q-1)}{2 n(1-q)}<0 .
\end{aligned}
$$

It is obvious that $b_{2 n+1}+b_{2 n+2}+\cdots>0$ and $\lim _{n \rightarrow \infty}\left(a_{n} / b_{n}\right)=0$. From $(2(k+m)-1)(2(k+m))>$ $(k+m)^{2}, k, m \in \mathbb{N}$ we have

$$
\frac{a_{2 k-1}+a_{2 k}+\cdots}{b_{2 k-1}+b_{2 k}+\cdots}=\frac{2\left(\alpha_{k} / k^{2}+\alpha_{k+1} /(k+1)^{2}+\cdots\right)}{\alpha_{k} /(2 k(2 k-1))+\alpha_{k+1} /((2 k+1)(2 k+2))+\cdots}>2 \quad \text { for } k \geq 1 .
$$

Thus $\lim _{n \rightarrow \infty}\left(a_{n}+a_{n+1}+\cdots\right) /\left(b_{n}+b_{n+1}+\cdots\right) \neq 0$.

Lemma 2.6. Let $\sum_{n=1}^{\infty} a_{n}, \sum_{n=1}^{\infty} b_{n}$ be convergent real series with nonzero terms. Let $b_{n}+b_{n+1}+\cdots \neq 0$ for all $n \in \mathbb{N}$. Let $l_{i}(a)=\liminf _{n \rightarrow \infty}\left|1+a_{n+1} / a_{n}+a_{n+2} / a_{n}+\cdots\right|, l_{s}(a)=\lim \sup _{n \rightarrow \infty} \mid 1+a_{n+1} / a_{n}+$ $a_{n+2} / a_{n}+\cdots\left|, l_{i}(b)=\liminf _{n \rightarrow \infty}\right| 1+b_{n+1} / b_{n}+b_{n+2} / b_{n}+\cdots\left|, l_{s}(b)=\lim \sup _{n \rightarrow \infty}\right| 1+b_{n+1} / b_{n}+$ $b_{n+2} / b_{n}+\cdots \mid$, then

(1) if $l_{s}(a)<\infty, l_{i}(b)>0, \lim _{n \rightarrow \infty}\left(a_{n} / b_{n}\right)=0$, then $\lim _{n \rightarrow \infty}\left(a_{n}+a_{n+1}+\cdots\right) /\left(b_{n}+b_{n+1}+\right.$ $\cdots)=0$,

(2) if $a_{n}+a_{n+1}+\cdots \neq 0$ for all $n \in \mathbb{N}, l_{i}(a)>0, l_{s}(b)<\infty, \lim _{n \rightarrow \infty}\left(a_{n}+a_{n+1}+\cdots\right) /\left(b_{n}+\right.$ $\left.b_{n+1}+\cdots\right)=0$, then $\lim _{n \rightarrow \infty}\left(a_{n} / b_{n}\right)=0$,

(3) if $a_{n}+a_{n+1}+\cdots \neq 0$ for all $n \in \mathbb{N}, 0<l_{i}(a), l_{s}(a)<\infty, 0<l_{i}(b), l_{s}(b)<\infty$, then $\lim _{n \rightarrow \infty}\left(a_{n} / b_{n}\right)=0$ if and only if $\lim _{n \rightarrow \infty}\left(a_{n}+a_{n+1}+\cdots\right) /\left(b_{n}+b_{n+1}+\cdots\right)=0$. 
Proof. For every $n \in \mathbb{N}$, we have

$$
\begin{aligned}
\left|\frac{a_{n}+a_{n+1}+\cdots}{b_{n}+b_{n+1}+\cdots}\right| & =\frac{\left|1+a_{n+1} / a_{n}+a_{n+2} / a_{n}+\cdots\right|}{\left|b_{n} / a_{n}+b_{n+1} / a_{n}+b_{n+2} / a_{n}+\cdots\right|} \\
& =\frac{\left|1+a_{n+1} / a_{n}+a_{n+2} / a_{n}+\cdots\right|}{\left|b_{n} / a_{n}\right|\left|1+b_{n+1} / b_{n}+b_{n+2} / b_{n}+\cdots\right|} \\
& =\frac{\left|a_{n} / b_{n}\right|\left|1+a_{n+1} / a_{n}+a_{n+2} / a_{n}+a_{n+3} / a_{n}+\cdots\right|}{\left|1+b_{n+1} / b_{n}+b_{n+2} / b_{n}+b_{n+3} / b_{n}+\cdots\right|} .
\end{aligned}
$$

From this follows our assertion.

Remark 2.7. The condition $\lim \inf _{n \rightarrow \infty}\left|1+b_{n+1} / b_{n}+b_{n+2} / b_{n}+\cdots\right|>0$ can be satisfied, for example, if limsup $\sup _{n \rightarrow \infty}\left|b_{n+1} / b_{n}\right|<1 / 2$. In fact, if $\limsup _{n \rightarrow \infty}\left|b_{n+1} / b_{n}\right|<r<1 / 2$. then there exists $n_{0} \in \mathbb{N}$ such that for every $n>n_{0}$, we have

$$
\begin{aligned}
\left|\frac{b_{n+1}}{b_{n}}+\frac{b_{n+2}}{b_{n}}+\frac{b_{n+3}}{b_{n}}+\cdots\right| & \leq\left|\frac{b_{n+1}}{b_{n}}\right|+\left|\frac{b_{n+2}}{b_{n+1}}\right|\left|\frac{b_{n+1}}{b_{n}}\right|+\left|\frac{b_{n+3}}{b_{n+2}}\right|\left|\frac{b_{n+2}}{b_{n+1}}\right|\left|\frac{b_{n+1}}{b_{n}}\right|+\cdots \\
& \leq r+r^{2}+r^{3}+\cdots=\frac{r}{1-r}
\end{aligned}
$$

and thus $\left|1+b_{n+1} / b_{n}+b_{n+2} / b_{n}+\cdots\right| \geq(1-2 r) /(1-r)>0$. The condition limsup $\sup _{n \rightarrow \infty} \mid 1+$ $a_{n+1} / a_{n}+a_{n+2} / a_{n}+\cdots \mid<\infty$ can be satisfied, for example, if $\limsup _{n \rightarrow \infty}\left|a_{n+1} / a_{n}\right|<1$. Indeed, if $\lim \sup _{n \rightarrow \infty}\left|a_{n+1} / a_{n}\right|=\alpha<1$, then there exists $n_{0} \in \mathbb{N}$ such that for every $n>n_{0}$, we have

$$
\begin{aligned}
\left|1+\frac{a_{n+1}}{a_{n}}+\frac{a_{n+2}}{a_{n}}+\cdots\right| & =\left|1+\frac{a_{n+1}}{a_{n}}+\frac{a_{n+2}}{a_{n+1}} \frac{a_{n+1}}{a_{n}}+\frac{a_{n+3}}{a_{n+2}} \frac{a_{n+2}}{a_{n+1}} \frac{a_{n+1}}{a_{n}}+\cdots\right| \\
& \leq 1+\beta+\beta^{2}+\beta^{3}+\cdots=\frac{1}{1-\beta}<\infty, \quad \text { where } \alpha<\beta<1 .
\end{aligned}
$$

Conversely, from the condition $\liminf _{n \rightarrow \infty}\left|1+b_{n+1} / b_{n}+b_{n+2} / b_{n}+\cdots\right|>0$ need not follow the condition $\lim \sup _{n \rightarrow \infty}\left|\left(b_{n+1} / b_{n}\right)\right|<1 / 2$. For example, if we put $a_{1} \neq 0, a_{2 n}=1 / 2 n$, $a_{2 n+1}=-1 / 2 n, n=1,2, \ldots$, then $\sum_{n=1}^{\infty} a_{n}$ is convergent series and $\limsup _{n \rightarrow \infty}\left|a_{n+1} / a_{n}\right|=1$, $\lim \sup _{n \rightarrow \infty}\left|1+a_{n+1} / a_{n}+a_{n+2} / a_{n}+\cdots\right|=1$.

\section{3. $\tau$-convergent series}

Definition 3.1 (see [10]). We say that a sequence $\left\{a_{n} ; n \in \mathbb{N}\right\}$ has $\tau$-limit a real number $L$ and we write $\tau$ - $\lim _{n \rightarrow \infty} a_{n}=L$, if for each $\varepsilon>0$ the set $A(\varepsilon)=\left\{n ;\left|a_{n}-L\right| \geq \varepsilon\right\}$ belongs to the ideal $\tau$, where $\tau$ is an admissible ideal of subsets of $\mathbb{N}$ which is additive (if $A, B \in \tau$, then $A \cup B \in \tau$ ), hereditary (if $B \subset A \in \tau$, then $B \in \tau$ ), containing all singletons and not containing $\mathbb{N}$.

We denote by $\tau_{f}$ the ideal of all finite subsets of $\mathbb{N}$.

Definition 3.2 (see [10]). We say that $\sum_{n=1}^{\infty} a_{n} \tau$-converges to a real number $L$ and we write $\tau-\sum_{n=1}^{\infty} a_{n}=L$ if for each $\varepsilon>0$ the set $A(\varepsilon)=\left\{n ;\left|\sum_{k=1}^{n} a_{k}-L\right| \geq \varepsilon\right\}$ belongs to the ideal $\tau$, where $\tau$ is an admissible ideal of subsets of $\mathbb{N}$. 
Definition 3.3. Let $\sum_{n=1}^{\infty} a_{n}, \sum_{n=1}^{\infty} b_{n}$ be $\tau$-convergent real series with nonzero terms such that $b_{n}+$ $b_{n+1}+\cdots \neq 0, n \in \mathbb{N}$. A series $\sum_{n=1}^{\infty} a_{n}$ is called $\tau$-faster convergent than $\sum_{n=1}^{\infty} b_{n}$ if $\tau-\lim _{n \rightarrow \infty}\left(a_{n+1}+\right.$ $\left.a_{n+2}+\cdots\right) /\left(b_{n+1}+b_{n+2}+\cdots\right)=0$.

Definition 3.4 (see [9]). Let $\tau$ be an admissible ideal of subsets of $\mathbb{N}$. A number $x \in R$ is said to be a $\tau$-cluster point of $\sum_{n=1}^{\infty} x_{n}$ if for each $\varepsilon>0$ the set $\left\{n \in \mathbb{N} ;\left|\sum_{k=1}^{n} x_{k}-x\right|<\varepsilon\right\}$ is not from $\tau$.

Remark 3.5. Of course, if $\tau$ is an admissible ideal of subsets of $\mathbb{N}$, by $\tau$-cluster point of a real sequence $\left\{x_{n} ; n \in \mathbb{N}\right\}$, we mean a number $x \in \mathbb{R}$, where for each $\varepsilon>0$ the set $\left\{n \in \mathbb{N} ;\left|x_{n}-x\right|<\varepsilon\right\}$ is not from $\tau$. Moreover, we say that $\infty(-\infty)$ is the $\tau$-cluster point of a real sequence $\left\{x_{n} ; n \in \mathbb{N}\right\}$ if for each $c>0(c<0)$ the set $\left\{n \in \mathbb{N} ; x_{n}>c\right\}\left(\left\{n \in \mathbb{N} ; x_{n}<c\right\}\right)$ is not from $\tau$.

Remark 3.6. If $\left\{x_{n} ; n \in \mathbb{N}\right\}$ is a real sequence, $\tau$ is an admissible ideal, and $X=\{x$; $x$ is a $\tau$-cluster point of $\left.\left\{x_{n} ; n \in \mathbb{N}\right\}\right\}$, then $X \neq \varnothing$., Indeed, if $\left\{x_{n} ; n \in \mathbb{N}\right\}$ is bounded, then by [8] there exists a $\tau$-cluster point of $\left\{x_{n} ; n \in \mathbb{N}\right\}$. If $\left\{x_{n} ; n \in \mathbb{N}\right\}$ is not bounded, then either $+\infty$ or $-\infty$ is $\tau$-cluster point of $\left\{x_{n} ; n \in \mathbb{N}\right\}$ according to Remark 3.5 or there exists $l \in \mathbb{R}$ such that $\left\{n \in \mathbb{N} ;\left|x_{n}\right|>l\right\} \in \tau$. If for some $l \in \mathbb{R}\left\{n \in \mathbb{N} ;\left|x_{n}\right|>l\right\} \in \tau$, then $K_{l}=\left\{n \in \mathbb{N} ;\left|x_{n}\right| \leq l\right\} \notin \tau$. Consider the ideal $v=\left\{K_{l} \cap A ; A \in \tau\right\}$. Because $\left\{x_{n} ; n \in K_{l}\right\}$ is a bounded set, there exists $x^{\star} \in \mathbb{R},\left|x^{\star}\right| \leq l$ such that $x^{\star}$ is a $v$-cluster point of $\left\{x_{n} ; n \in K_{l}\right\}$. Let $\varepsilon>0$. Since $x^{\star}$ is a $v$-cluster point of $\left\{x_{n} ; n \in K_{l}\right\}$, then the set $\left\{n \in K_{l} ;\left|x_{n}-x^{\star}\right|<\varepsilon\right\} \notin v$ and then also is not from $\tau$. Since $\tau$ has a hereditary property, then $\left\{n \in \mathbb{N} ;\left|x_{n}-x^{\star}\right|<\varepsilon\right\} \notin \tau$. So $x^{\star}$ is a cluster point of $\left\{x_{n} ; n \in \mathbb{N}\right\}$.

Definition 3.7. Let $\tau$ be an admissible ideal of subsets of $\mathbb{N}$. Let $\sum_{n=1}^{\infty} x_{n}$ be an infinite series of real numbers and let $X=\left\{x \in \mathbb{R} ; x\right.$ is a $\tau$-cluster point of $\left.\sum_{n=1}^{\infty} x_{n}\right\}$. If $X$ is bounded, then $s=\sup X(s=\inf X)$ is said to be a $\tau-\lim \sup _{n \rightarrow \infty} \sum_{n=1}^{\infty} x_{n}\left(\tau-\liminf \operatorname{in}_{n \rightarrow \infty} \sum_{n=1}^{\infty} x_{n}\right)$. If $\sup X=\infty$ or $\infty$ is $\tau$-cluster point (inf $X=-\infty$ or $-\infty$ is $\tau$-cluster point), then $\tau$-lim $\sup _{n \rightarrow \infty} \sum_{n=1}^{\infty} x_{n}=\infty$ $\left(\tau\right.$-liminf $\left.\operatorname{in}_{n \rightarrow \infty} \sum_{n=1}^{\infty} x_{n}=-\infty\right)$.

Definition 3.8. We say that a sequence $\left\{x_{n} ; n \in \mathbb{R}\right\}$ is $\tau$-bounded above ( $\tau$-bounded bellow), if there exist $m \in \mathbb{R}$ such that $\left\{n \in \mathbb{N} ; x_{n}>m\right\} \in \tau\left(\left\{n \in \mathbb{N} ; x_{n}<m\right\} \in \tau\right)$ and is $\tau$-bounded if it is $\tau$-bounded above and below simultaneously.

It is obvious that if $\sum_{n=1}^{\infty} a_{n}$ is faster convergent than $\sum_{n=1}^{\infty} b_{n}$, then $\sum_{n=1}^{\infty} a_{n}$ is $\tau$-faster convergent, the $\sum_{n=1}^{\infty} b_{n}$. Generally, from the fact that $\sum_{n=1}^{\infty} a_{n}$ is $\tau$-faster convergent, $\sum_{n=1}^{\infty} b_{n}$ does not hold that $\sum_{n=1}^{\infty} a_{n}$ is faster convergent than $\sum_{n=1}^{\infty} b_{n}$.

It is obvious that for $\tau$-convergent series where $\tau$ has the property $\mathrm{p} 1$ :

if $M \in \tau, \quad$ then $M+1=\{n+1 ; n \in M \cap N\} \in \tau, \quad M-1=\{n-1>0 ; n \in M \cap N\} \in \tau$

( $\tau_{f}$ or $\tau_{s t}=\{A \subset N$; asymptotic density $(A)=0\}$ have 1 1), we obtain similar lemma as Lemma 2.6.

Lemma 3.9. Let $\tau$ be an admissible ideal of subsets of $\mathbb{N}$ with property $\mathrm{p} 1$. Let $\sum_{n=1}^{\infty} a_{n}, \sum_{n=1}^{\infty} b_{n}$ be $\tau$-convergent real series with nonzero terms. Let $b_{n}+b_{n+1}+\cdots \neq 0$, for all $n \in \mathbb{N}$. Let

$$
\begin{array}{ll}
t_{i}(a)=\tau-\liminf _{n \rightarrow \infty}\left|1+\frac{a_{n+1}}{a_{n}}+\frac{a_{n+2}}{a_{n}}+\cdots\right|, & t_{s}(a)=\tau-\limsup _{n \rightarrow \infty}\left|1+\frac{a_{n+1}}{a_{n}}+\frac{a_{n+2}}{a_{n}}+\cdots\right|, \\
t_{i}(b)=\tau-\liminf _{n \rightarrow \infty}\left|1+\frac{b_{n+1}}{b_{n}}+\frac{b_{n+2}}{b_{n}}+\cdots\right|, & t_{s}(b)=\tau-\limsup \left|1+\frac{b_{n+1}}{b_{n}}+\frac{b_{n+2}}{b_{n}}+\cdots\right| .
\end{array}
$$


Then

(1) if $t_{s}(a)<\infty, t_{i}(b)>0, \tau-\lim _{n \rightarrow \infty}\left(a_{n} / b_{n}\right)=0$, then $\tau-\lim _{n \rightarrow \infty}\left(a_{n}+a_{n+1}+\cdots\right) /\left(b_{n}+b_{n+1}+\right.$ $\cdots)=0$,

(2) if $a_{n}+a_{n+1}+\cdots \neq 0$ for all $n \in \mathbb{N}, t_{i}(a)>0, t_{s}(b)<\infty, \tau-\lim _{n \rightarrow \infty}\left(a_{n}+a_{n+1}+\cdots\right) /\left(b_{n}+\right.$ $\left.b_{n+1}+\cdots\right)=0$, then $\tau$ - $\lim _{n \rightarrow \infty}\left(a_{n} / b_{n}\right)=0$,

(3) if $a_{n}+a_{n+1}+\cdots \neq 0$ for all $n \in \mathbb{N}, 0<t_{i}(a), t_{s}(a)<\infty, 0<t_{i}(b), t_{s}(b)<\infty$, then $\tau-\lim _{n \rightarrow \infty}\left(a_{n} / b_{n}\right)=0$, if and only if $\tau-\lim _{n \rightarrow \infty}\left(a_{n}+a_{n+1}+\cdots\right) /\left(b_{n}+b_{n+1}+\cdots\right)=0$.

Proof. First, we note that if $\tau$-limsup $\sup _{n \rightarrow \infty} x_{n}<\infty$, then sequence $\left\{x_{n} ; n \in \mathbb{N}\right\}$ is $\tau$-bounded above (similarly for $\tau$-liminf). Next, we show that if $\left\{a_{n} ; n \in \mathbb{N}\right\}$ is a $\tau$-convergent sequence to 0 and $\left\{b_{n} ; n \in \mathbb{N}\right\}$ is a $\tau$-bounded sequence, then $\left\{a_{n} b_{n}: n \in \mathbb{N}\right\}$ is a $\tau$-convergent sequence to 0 . There exist $k \in \mathbb{R}$ such that the set $N_{b}=\left\{n ;\left|b_{n}\right| \geq k\right\}$ is from $\tau$. Let $\varepsilon>0$. The set $N_{a}(\varepsilon)=\left\{n ;\left|a_{n}\right| \geq \varepsilon / k\right\}$ is from $\tau$. If $n \in \mathbb{N} \backslash\left(N_{a}(\varepsilon) \cup N_{b}\right)$, then $\left|a_{n}\right|<\varepsilon / K$ and $\left|b_{n}\right|<k$ and so $\left|a_{n} b_{n}\right|<\varepsilon$. From this and from properties of ideal $\tau$, follows that $\left\{a_{n} b_{n}: n \in \mathbb{N}\right\} \tau$-converges to 0 . Then the proof follows from Lemma 2.6.

The following examples show that we cannot replace lim by $\tau$-lim in Lemmas 1.2, 2.1, and 2.2.

Example 3.10. Let $\sum_{n=1}^{\infty} b_{n}$ be a convergent series with positive terms such that $\sum_{n=1}^{\infty}\left(b_{n}+b_{n+1}+\right.$ $b_{n+2}+\cdots$ ) is convergent (e.g., $\sum_{n=1}^{\infty} b_{n}=\sum_{n=1}^{\infty} \alpha^{n-1}, 0<\alpha<1$ ). Let $M=\left\{i_{j} ; 1<i_{j}<i_{j+1}, j \in \mathbb{N}\right\}$, $M \in \tau$ be an infinite subset of $\mathbb{N}$, where $\tau$ is an arbitrary admissible ideal with property p1 different from $\tau_{f}$. We define a real series $\sum_{n=1}^{\infty} a_{n}$ in the following way:

$$
a_{n}= \begin{cases}\frac{1}{n} b_{n}, & n>1, n \neq i_{j}, i_{j} \in M, j=2,3, \ldots \\ b_{i_{j-1}}+b_{i_{j-1}+1}+\cdots & n \in M \backslash\left\{i_{1}\right\}, \\ c, & n=1,\end{cases}
$$

where $c$ be a real number such that $\sum_{n=1}^{\infty} a_{n}=\sum_{n=1}^{\infty} b_{n}$. We obtain $\tau$ - $\lim _{n \rightarrow \infty}\left(a_{n} / b_{n}\right)=0,\left(a_{n}+\right.$ $\left.a_{n+1}+\cdots\right) /\left(b_{n}+b_{n+1}+\cdots\right)>1$ for all $n \geq i_{2}$.

Example 3.11. Let $M=\left\{n_{j} ; n_{1}=1, n_{j}<n_{j+1}, n_{j+1}-n_{j} \geq 2, j \in \mathbb{N}\right\}, M \in \tau$ be an infinite subset of $\mathbb{N}$, where $\tau$ is an arbitrary admissible ideal with property p1 different from $\tau_{f}$. Let $\left\{B_{n_{j}}\right\}_{j=1}^{\infty}$ be a sequence of positive real numbers such that $B_{n_{j+1}}<B_{n_{j}}<m B_{n_{j+1}}, \lim _{j \rightarrow \infty} B_{n_{j}}=0$, where $m>1$. Put $A_{k}=B_{k} / 2^{k}, k \in M$. Let $A_{k}, B_{k}$ for $k \notin M$ be defined as follows: if $n_{j}<k<n_{j+1}$, where $j \in \mathbb{N}$ we put $\varepsilon_{n_{j}}=\left(A_{n_{j}}-A_{n_{j+1}}\right) /\left(n_{j+1}-n_{j}\right), A_{k}=A_{n_{j}}-\left(k-n_{j}\right) \varepsilon_{n_{j}}, B_{k}=B_{n_{j}}-\left(k-n_{j}\right) \varepsilon_{n_{j}}$. For $k \notin M$, we have $A_{k} / B_{k}<A_{n_{j}} / B_{n_{j+1}}=B_{n_{j}} / 2^{n_{j}} B_{n_{j+1}}<m / 2^{n_{j}}$, where $n_{j}<k<n_{j+1}$. From this and from the definition $A_{k}, k \in M$, it follows $\lim _{k \rightarrow \infty}\left(A_{k} / B_{k}\right)=0$, hence $\tau$ - $\lim _{k \rightarrow \infty}\left(A_{k} / B_{k}\right)=0$. It is obvious that $\left(A_{k}-A_{k+1}\right) /\left(B_{k}-B_{k+1}\right)=1$ for $k \neq n_{j}-1, j>1$. Let $A_{0}>A_{1}$ and $B_{0}>B_{1}$ be real numbers. Put $a_{n}=A_{n-1}-A_{n}, b_{n}=B_{n-1}-B_{n}, n \in \mathbb{N}$. The series $\sum_{n=1}^{\infty} a_{n}, \sum_{n=1}^{\infty} b_{n}$ are convergent with positive terms and $\tau-\lim _{n \rightarrow \infty}\left(a_{n}+a_{n+1}+\cdots\right) /\left(b_{n}+b_{n+1}+\cdots\right)=0, \tau-\lim _{n \rightarrow \infty}\left(a_{n} / b_{n}\right)=1$.

Remark 3.12. If $\tau$ have not the property p1, we get similar lemma as Lemma 3.9, but with shift indices of given series. (In general, it does not hold that if $\tau-\lim _{n \rightarrow \infty} a_{n}=a$, then $\tau$ - $\lim _{n \rightarrow \infty} a_{n+1}=$ $a$, and so on.) 


\section{Kummer series}

In [7], is showed: if $\sum_{n=1}^{\infty} a_{n}$ is a convergent real series with positive terms and with an unknown sum $a$ and $\sum_{n=1}^{\infty} c_{n}$ is a convergent real series with positive terms and with a known sum $c$, then if $\lim _{n \rightarrow \infty}\left(a_{n} / c_{n}\right)=p>0$ the Kummer series $\sum_{n=1}^{\infty} b_{n}$, where $b_{1}=a_{1}+p\left(c-c_{1}\right)$ and $b_{n}=a_{n}-p c_{n}$ for $n \geq 2$, has the same sum as $\sum_{n=1}^{\infty} a_{n}$ and if $b_{n}>0$ is a faster convergent series then $\sum_{n=1}^{\infty} a_{n}$.

In the following lemma, we prove by using Lemma 2.6 the faster convergence of the Kummer series $\sum_{n=1}^{\infty} b_{n}$ to the unknown sum of $\sum_{n=1}^{\infty} a_{n}$ without conditions of positivity of $p$ and terms of $\sum_{n=1}^{\infty} a_{n}, \sum_{n=1}^{\infty} b_{n}, \sum_{n=1}^{\infty} c_{n}$.

Lemma 4.1. Let $\sum_{n=1}^{\infty} a_{n}$ be a convergent real series with nonzero terms and with the sum a. Let $\liminf _{n \rightarrow \infty}\left|1+a_{n+1} / a_{n}+a_{n+2} / a_{n}+\cdots\right|>0$. Let $a_{n}+a_{n+1}+\cdots \neq 0$ for all $n \in \mathbb{N}$. Let $\sum_{n=1}^{\infty} c_{n}$ be a convergent series with nonzero terms and with the sum $c$. Let $\lim _{n \rightarrow \infty} a_{n} / c_{n}=p \neq 0$. If for the series $\sum_{n=1}^{\infty} b_{n}$, where $b_{1}=a_{1}+p\left(c-c_{1}\right)$ and $b_{n}=a_{n}-p c_{n}$, for $n \geq 2$, is $b_{n} \neq 0$, for $n \geq 2$, and $\lim \sup _{n \rightarrow \infty}\left|1+b_{n+1} / b_{n}+b_{n+2} / b_{n}+\cdots\right|<\infty$, then $\sum_{n=1}^{\infty} b_{n}=a$ and $\sum_{n=1}^{\infty} b_{n}$ is a faster convergent series than $\sum_{n=1}^{\infty} a_{n}$.

Proof. Since $\lim _{n \rightarrow \infty}\left(b_{n} / a_{n}\right)=0$, the proof follows from Lemma 2.6.

In the next example, we construct, by using of Remark 2.7 and Lemma 4.1, the Kummer series $\sum_{n=1}^{\infty} b_{n}$ faster convergent than the series $\sum_{n=1}^{\infty} a_{n}$ such that series $\sum_{n=1}^{\infty} a_{n}, \sum_{n=1}^{\infty} c_{n}, \sum_{n=1}^{\infty} b_{n}$ does not have positive terms.

Example 4.2. Let $\sum_{n=1}^{\infty} a_{n}=\sum_{n=1}^{\infty}(-1)^{n} /\left(4^{n}\left(3 n^{2}+\sqrt{ } n\right)\right)$, (the sum is unknown). It is evident that $\lim \sup _{n \rightarrow \infty}\left|a_{n+1} / a_{n}\right|=1 / 4<1 / 2$. Let $\sum_{n=1}^{\infty} c_{n}=\sum_{n=1}^{\infty}(-1)^{n} /\left(4^{n} n^{2}\right)$, then $\lim _{n \rightarrow \infty} a_{n} / c_{n}=1 / 3$ and from $\sum_{n=1}^{\infty}\left(x^{n} / n^{2}\right)=-\int_{0}^{x}(\ln (1-t) / t) d t, x \in(-1,1)$, it follows $\sum_{n=1}^{\infty}(-1)^{n} /\left(4^{n} n^{2}\right)=$ $\int_{-1 / 4}^{0}(\ln (1-t) / t) d t$ (the sum is known). The Kummer series is $\sum_{n=1}^{\infty} b_{n}=b_{1}+\sum_{n=2}^{\infty}\left(a_{n}-(1 / 3) c_{n}\right)$, where $\sum_{n=2}^{\infty} b_{n}=\sum_{n=2}^{\infty}(-1)^{n+1} /\left(4^{n} 3 n \sqrt{ } n\left(3 n^{2}+\sqrt{ } n\right)\right), b_{1}=1 / 48+1 / 3 \int_{-1 / 4}^{0}(\ln (1-t) / t) d t$. It is obvious that $\limsup _{n \rightarrow \infty}\left|b_{n+1} / b_{n}\right|=1 / 4<1$. Because $\sum_{n=1}^{\infty} a_{n}$ is series with alternating signs such that for all $n \in \mathbb{N}$ we have that $\left|a_{n+1}\right|<\left|a_{n}\right|$, we get for $n \in \mathbb{N} a_{2 n}+a_{2 n+1}+\cdots>$ $0, a_{2 n+1}+a_{2 n+2}+\cdots<0$. Hence $\sum_{n=1}^{\infty} b_{n}$ is a faster convergent series than $\sum_{n=1}^{\infty} a_{n}$ and it has the same sum.

\section{References}

[1] D. A. Smith and W. F. Ford, "Acceleration of linear and logarithmic convergence," SIAM Journal on Numerical Analysis, vol. 16, no. 2, pp. 223-240, 1979.

[2] T. A. Keagy and W. F. Ford, "Acceleration by subsequence transformations," Pacific Journal of Mathematics, vol. 132, no. 2, pp. 357-362, 1988.

[3] J. Wimp, "Some transformations of monotone sequences," Mathematics of Computation, vol. 26, no. 117, pp. 251-254, 1972.

[4] H. E. Salzer, "A simple method for summing certain slowly convergent series," Journal of Mathematics and Physics, vol. 33, pp. 356-359, 1955.

[5] D. F. Dawson, "Matrix summability over certain classes of sequences ordered with respect to rate of convergence," Pacific Journal of Mathematics, vol. 24, no. 1, pp. 51-56, 1968.

[6] B. C. Tripathy and M. Sen, "A note on rate of convergence of sequences and density of subsets of natural numbers," Italian Journal of Pure and Applied Mathematics, no. 17, pp. 151-158, 2005.

[7] T. Šalát, Nekonečné Rady, ACADEMIA Nakladatelství Československé Akademie, Prague, Czech Republic, 1974. 
[8] R. Filipów, I. Recław, N. Mrożek, and P. Szuca, "Ideal convergence of bounded sequences," Journal of Symbolic Logic, vol. 72, no. 2, pp. 501-512, 2007.

[9] P. Kostyrko, T. Šalát, and W. Wilczyński, "O-convergence," Real Analysis Exchange, vol. 26, no. 2, pp. 669-685, 2001.

[10] T. Šalát and V. Toma, "A classical Olivier's theorem and statistical convergence," Annales Mathématiques Blaise Pascal, vol. 10, no. 2, pp. 305-313, 2003.

[11] M. Dindoš, T. Šalát, and V. Toma, "Statistical convergence of infinite series," Czechoslovak Mathematical Journal, vol. 53(128), no. 4, pp. 989-1000, 2003.

[12] T. Šalát, "On statistically convergent sequences of real numbers," Mathematica Slovaca, vol. 30, no. 2, pp. 139-150, 1980.

[13] B. C. Tripathy, "On statistical convergence," Proceedings of the Estonian Academy of Sciences. Physics, Mathematics, vol. 47, no. 4, pp. 299-303, 1998.

[14] B. C. Tripathy, “On statistically convergent series," The Punjab University. Journal of Mathematics, vol. 32, pp. 1-8, 1999. 


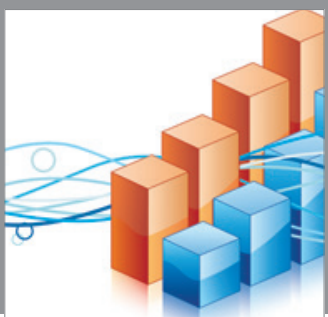

Advances in

Operations Research

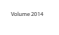

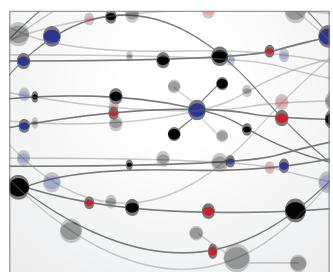

\section{The Scientific} World Journal
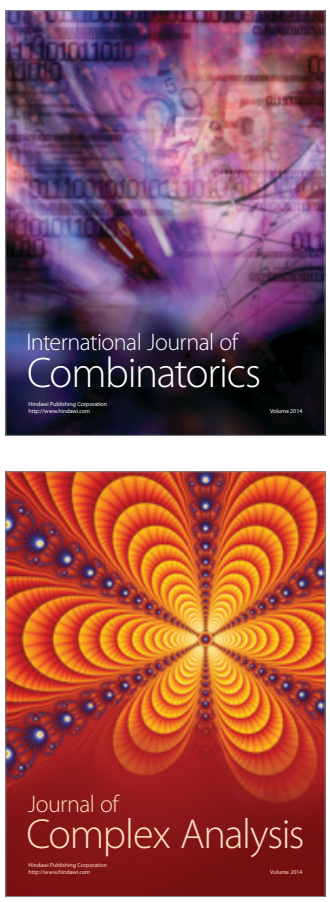

International Journal of

Mathematics and

Mathematical

Sciences
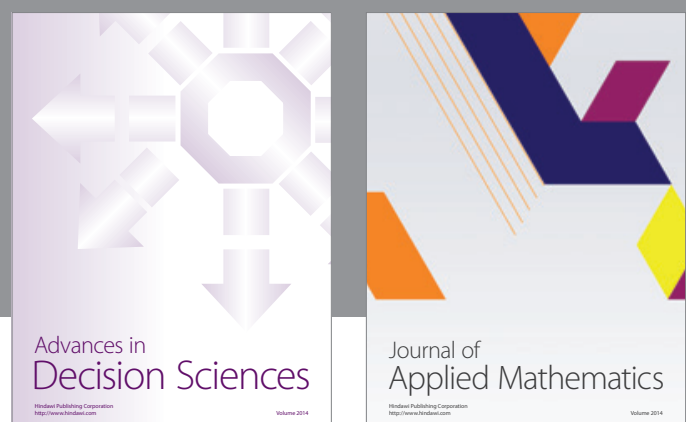

Journal of

Applied Mathematics
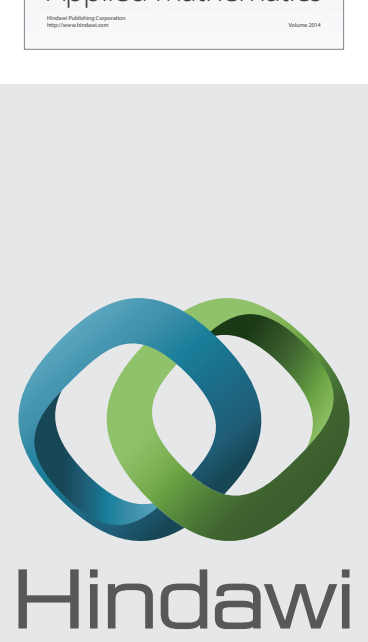

Submit your manuscripts at http://www.hindawi.com
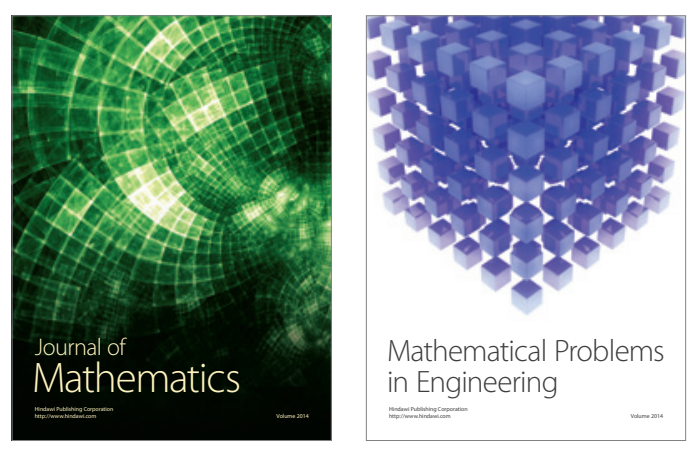

Mathematical Problems in Engineering
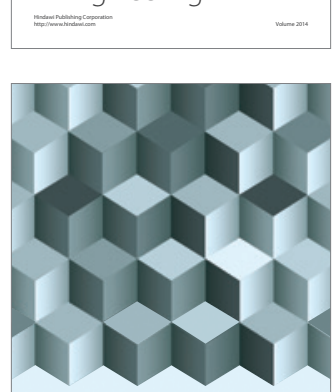

Journal of

Function Spaces
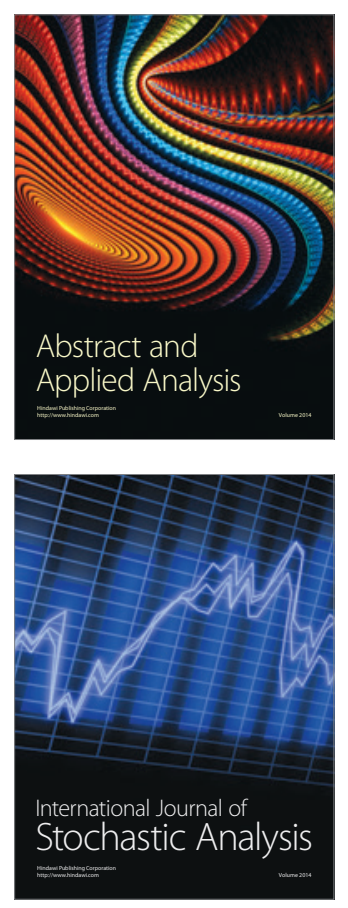

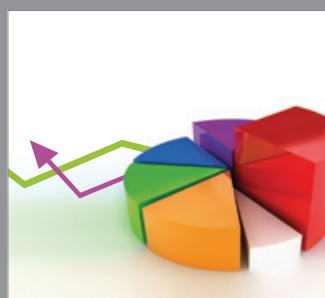

ournal of

Probability and Statistics

Promensencen
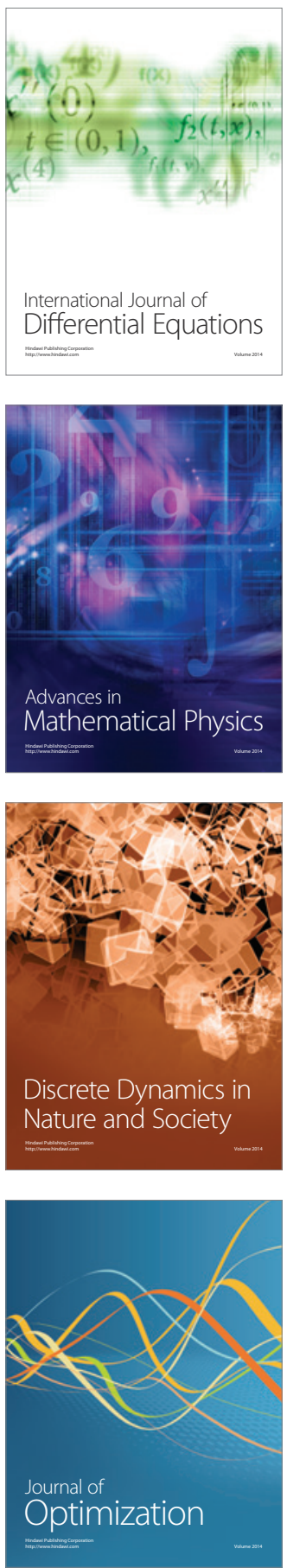